Georgian Mathematical Journal

Volume 8 (2001), Number 2, 389-400

\title{
ON STOCHASTIC DIFFERENTIAL EQUATIONS IN A CONFIGURATION SPACE
}

\author{
A. SKOROKHOD
}

\begin{abstract}
Infinite systems of stochastic differential equations for randomly perturbed particle systems with pairwise interaction are considered. It is proved that under some reasonable assumption on the potential function there exists a local weak solution to the system and it is weakly locally unique for a wide class of initial conditions.
\end{abstract}

2000 Mathematics Subject Classification: 60H10, 60H30.

Key words and phrases: Configuration space, stochastic differential equation, local weak solution.

\section{INTRODUCTION}

We consider a sequence of $R^{d}$-valued stochastic processes

$$
x_{k}(t), \quad k=1,2, \ldots,
$$

satisfying the system of stochastic differential equations of the form

$$
d x_{k}(t)=\sum_{i \neq k} a\left(x_{k}(t)-x_{i}(t)\right) d t+\sigma d w_{k}(t), \quad k=1,2, \ldots,
$$

where $a(x)=-U_{x}(x)$, and $U: R^{d} \rightarrow R$ is a smooth function for $|x|>0$, and $\sigma>0$ is a constant, $w_{k}(t), k=1,2, \ldots$, is a sequence of independent Wiener processes in $R^{d}$. System (1) describes the evolution of systems of pairwise interacting particles with the pairwise potential $U(x)$ which is perturbed by Wiener noises. The problem is to find conditions under which the system has a solution and this solution is unique.

Unperturbed systems were considered by many authors. We notice the recent articles of S. Albeverio, Yu. G. Kondratiev, and M. Röckner [1], [2] where a new powerful method for the investigation of unperturbed systems is proposed. Finite-dimensional perturbed systems were considered in my book [3] and my article [4]. The first general theorem on the existence and uniqueness of the solutions to infinite dimensional stochastic differential equations were obtained by Yu. L. Daletskii in [5]; he considered equations with smooth coefficients in a Hilbert space. The existence and uniqueness of the solution to system (1) for locally bounded smooth potentials and $d \leq 2$ was proved by J. Fritz in [6]. The main result of this article was published in [7] without a complete proof. 


\section{The Space $\Gamma$}

It is convenient to consider system (1) in the configuration space $\Gamma$ which is the set of locally finite counting measures $\gamma$ on the Borel $\sigma$-algebra $\mathcal{B}\left(R^{d}\right)$ of the space $R^{d}$. So a measure $\gamma \in \Gamma$ satisfies the condition: the support $S_{\gamma}$ of the measure $\gamma$ is a sequence of different points $\left\{x_{k}, k \in \mathcal{N}\right\}$ of $R^{d}$ for which

$$
\left|x_{k}\right| \rightarrow \infty, \quad \gamma(A)=\sum_{k} 1_{A}\left(x_{k}\right) .
$$

The topology in $\Gamma$ is generated by the weak convergence of measures: $\gamma_{n} \rightarrow \gamma_{0}$ if

$$
\int \phi(x) \gamma_{n}(d x) \rightarrow \int \phi(x) \gamma_{0}(d x)
$$

for $\phi \in \mathcal{C}_{f}$ where $\mathcal{C}_{f}$ is the set of continuous functions $\phi: R^{d} \rightarrow R$ with bounded supports.

We use the notation

$$
\langle\phi, \gamma\rangle=\int \phi(x) \gamma(d x), \quad \phi \in \mathcal{C}_{f}
$$

and

$$
\langle\Phi, \gamma \times \gamma\rangle=\int \Phi\left(x, x^{\prime}\right) \gamma(d x) \gamma\left(d x^{\prime}\right)-\int \Phi(x, x) \gamma(d x),
$$

where $\Phi:\left(R^{d}\right)^{2} \rightarrow R$ is a continuous function with a bounded support.

We rewrite system (1) for $\Gamma$-valued function $\gamma_{t}$ for which

$$
\langle\phi, \gamma\rangle=\sum_{k} \phi\left(x_{k}(t)\right), \quad \phi \in \mathcal{C}_{f} .
$$

Using the Itô's formula, and considering the function $a$ as a function of two variables $a\left(x-x^{\prime}\right)$, we obtain the relation

$$
\begin{aligned}
d\left\langle\phi, \gamma_{t}\right\rangle & =\left\langle\left(\phi^{\prime}, a\right), \gamma_{t} \times \gamma_{t}\right\rangle d t+\frac{\sigma^{2}}{2}\left\langle\Delta \phi, \gamma_{t}\right\rangle \\
& +\sum_{k} \sigma\left(\phi^{\prime}\left(x_{k}(t)\right), d w_{k}(t)\right), \quad \phi \in \mathcal{C}_{f}^{(2)},
\end{aligned}
$$

where $\Delta \phi(x)=\operatorname{Tr} \phi^{\prime \prime}(x)$, and $\mathcal{C}_{f}^{(2)}$ is the set of $\phi \in \mathcal{C}_{f}$ for which $\phi^{\prime}(x)$ and $\phi^{\prime \prime}(x)$ are continuous bounded functions.

A weak solution to equation (1). A $\Gamma$-valued stochastic process $\gamma_{t}(\omega)$ is called a weak solution to system (2) if, for all $\phi \in \mathcal{C}_{f}^{(2)}$, the stochastic process

$$
\mu_{\phi}(\omega, t)=\left\langle\phi, \gamma_{t}\right\rangle-\int_{0}^{t}\left[\left\langle\left(\phi^{\prime}, a\right), \gamma_{s} \times \gamma_{s}\right\rangle+\frac{\sigma^{2}}{2}\left\langle\Delta \phi, \gamma_{s}\right\rangle\right] d s
$$

is a martingale with respect to the filtration $\left(\mathcal{F}_{t}\right)_{t \geq 0}$, where $\mathcal{F}_{t}=\sigma\left(\gamma_{s}, s \leq t\right)$, and the square characteristic of the martingale is

$$
\left\langle\mu_{\phi}, \mu_{\phi}\right\rangle_{t}=\sigma^{2} \int_{0}^{t}\left\langle\left(\phi^{\prime}, \phi^{\prime}\right), \gamma_{s}\right\rangle d s .
$$


If $\gamma_{t}(\omega)$ is a weak solution to system (2) and

$$
\left\langle\phi, \gamma_{t}(\omega)\right\rangle=\sum \phi\left(x_{k}(t)\right)
$$

for all $\phi \in \mathcal{C}_{f}$, then the sequence $\left\{x_{k}(t), k \in \mathcal{N}\right\}$ is a weak solution to system (1).

Weak uniqueness. Let $\gamma_{0} \in \Gamma$. System (2) has a unique weak solution with the initial value $\gamma_{0}$ if for any pair of weak solutions to system $(2) \gamma_{t}^{1}(\omega)$ and $\gamma_{t}^{2}(\omega)$ satisfying the condition $\gamma_{0}^{1}=\gamma_{0}^{2}=\gamma_{0}$, the following relations are fulfilled:

$$
E \Phi\left(\xi_{11}^{1}, \ldots, \xi_{1 m}^{1}, \ldots, \xi_{l 1}^{1}, \ldots, \xi_{l m}^{1}\right)=E \Phi\left(\xi_{11}^{2}, \ldots, \xi_{1 m}^{2}, \ldots, \xi_{l 1}^{2}, \ldots, \xi_{l m}^{2}\right),
$$

where $\Phi\left(y_{11}, \ldots, y_{l m}\right)$ is a continuous bounded function on $R^{l m}$ and,

$$
\begin{gathered}
\xi_{i j}^{k}=\left\langle\phi_{i}, \gamma_{t_{j}}^{k}(\omega)\right\rangle, \quad k=1,2, \quad i=1, \ldots, l, \quad j=1, \ldots, m, \\
\phi \in \mathcal{C}_{f}, \quad t_{1}, \ldots, t_{m} \in R_{+} .
\end{gathered}
$$

This means that the distribution of the stochastic process $\gamma_{t}^{k}$ does not depend on $k$, i.e., the distribution of the weak solution to system (2) is unique if it exists.

Local weak solutions. Let $\gamma_{t}(\omega)$ be a continuous $\Gamma$-valued stochastic process, and $\left(\mathcal{F}_{t}\right)_{t>0}$ be the filtration generated by it. $\gamma_{t}(\omega)$. is called a local weak solution to system (2) if there exists a stopping time $\tau$ with respect to the filtration $\left(\mathcal{F}_{t}\right)_{t \geq 0}$ for which $P\{\tau>0\}=1$ and the stochastic process $\mu_{\phi}(\omega, t)$ which is determined by relation (3) is a martingale for $t<\tau$ with the square characteristic given by equality (4).

Local weak uniqueness. Let $\gamma_{0} \in \Gamma$. System (2) has a locally unique weak solution with the initial value $\gamma_{0}$ if, for any pair of locally weak solutions to system (2) $\gamma_{t}^{1}$ and $\gamma_{t}^{2}$ satisfying the condition $\gamma_{0}^{1}=\gamma_{0}^{2}=\gamma_{0}$, relation (5) is fulfilled for

$$
\xi_{i j}^{k}=\left\langle\phi_{i}, \gamma_{t_{j}}^{k}(\omega)\right\rangle I_{\left\{t_{j}<\tau^{k}\right\}},
$$

where $\tau^{k}, k=1,2$, are stopping times with respect to the filtration $\left(\mathcal{F}_{t}^{k}\right)_{t \geq 0}$ generated by the stochastic process $\gamma_{t}^{k}(\omega)$.

Compacts in $\Gamma$. For any $\gamma \in \Gamma$ and a continuous decreasing function $\lambda(t)$ : $(0, \infty) \rightarrow R_{+}$for which $\lambda(0+)=+\infty, \lambda(+\infty)>0$ there exists a continuous decreasing function $\Phi(t):[0, \infty) \rightarrow R_{+}$with $\Phi(+\infty)=0$ such that

$$
\iint \Phi(|x|) \Phi\left(\left|x^{\prime}\right|\right) \lambda\left(\left|x-x^{\prime}\right|\right) 1_{\left\{x \neq x^{\prime}\right\}} \gamma(d x) \gamma\left(d x^{\prime}\right)<\infty
$$

Denote

$$
\Phi_{\lambda}\left(x, x^{\prime}\right)=\Phi(|x|) \Phi\left(\left|x^{\prime}\right|\right) \lambda\left(\left|x-x^{\prime}\right|\right) .
$$

For any compact set $K$ from $\Gamma$ and any fanction $\lambda$ satisfying the conditions mentioned before there exists a function of the form given by relation (7) for which

$$
\sup _{\gamma \in K}\left\langle\Phi_{\lambda}, \gamma \times \gamma\right\rangle<\infty
$$


Note that the set

$$
\left\{\gamma:\left\langle\Phi_{\lambda}, \gamma \times \gamma\right\rangle \leq c\right\}
$$

is a compact in $\Gamma$ for any $\Phi_{\lambda}$ of form $(7)$ and $c>0$. Denote by $\Gamma_{\Phi, \lambda}$ the set of those $\gamma \in \Gamma$ for which relation (6) is fulfilled. Set

$d_{\Phi, \lambda}\left(\gamma_{1}, \gamma_{2}\right)=\sup \left\{\left|\left\langle\phi \Phi, \gamma_{1}\right\rangle-\left\langle\phi \Phi, \gamma_{2}\right\rangle: \phi \in \operatorname{Lip}^{1}\right|\right\}+\left|\left\langle\Phi_{\lambda}, \gamma_{1} \times \gamma_{1}\right\rangle-\left\langle\Phi_{\lambda}, \gamma_{2} \times \gamma_{2}\right\rangle\right|$,

where

$$
\operatorname{Lip}^{1}=\left\{\phi \in C_{f}: \sup _{x}|\phi(x)| \leq 1, \quad \sup _{x, x^{\prime}} \frac{\left|\phi(x)-\phi\left(x^{\prime}\right)\right|}{\left|x-x^{\prime}\right|} \leq 1\right\} .
$$

$\Gamma_{\Phi, \lambda}$ with the distance $d_{\Phi, \lambda}$ is a separable locally compact space.

\section{An Extension of Girsanov's Formula}

Assume that the potential function $U(x)$ satisfies the condition

(PC) $U(x)=u(|x|)$ where the function $u:(0, \infty) \rightarrow R$ is continuous, it has continuous derivatives $u^{\prime}, u^{\prime \prime}$, there exists a constant $r>0$ for which $u(t)=0$ for $t>r$, and

$$
\int t^{d-1}|u(t)| d t<\infty
$$

Free particle processes. Let a measure $\gamma_{0}$ satisfy the condition (IC)

$$
\left\langle\phi_{\delta}, \gamma_{0}\right\rangle<\infty, \quad \phi_{\delta}(x)=\exp \left\{-\delta|x|^{2}\right\} .
$$

Introduce $\Gamma$-valued stochastic processes by the relation

$$
\left\langle\phi, \gamma_{t}^{*}\left(\gamma_{0}, \omega\right)\right\rangle=\sum_{k} \phi\left(x_{k}^{0}+\sigma w_{k}(t)\right)
$$

where

$$
\sum_{k} \phi\left(x_{k}^{0}\right)=\left\langle\phi, \gamma_{0}\right\rangle
$$

It is easy to check that $\gamma_{t}^{*}\left(\gamma_{0}, \omega\right)$ is a continuous $\Gamma$-valued stochastic process if $\gamma_{0} \in \Gamma^{0}$, where $\Gamma^{0}$ is the set of finite measures from $\Gamma$. There exist functions $\Phi, \lambda$ for which $P\left\{\gamma_{t}^{*}\left(\gamma_{0}, \omega\right) \in \Gamma_{\Phi, \lambda}\right\}=1$ for all $t>0$. The stochastic process $\gamma_{t}^{*}\left(\gamma_{0}, \omega\right)$ is continuous in the space $\Gamma_{\Phi, \lambda}$, and for any $t_{0}>0$ the function

$$
\operatorname{EF}\left(\gamma^{*}\left(\gamma_{0}, \omega\right)\right)
$$

is a continuous function in $\gamma_{0} \in \Gamma_{\Phi, \lambda}$ if $F$ is a bounded continuous function on $C_{\left[0, t_{0}\right]}\left(\Gamma_{\Phi, \lambda}\right)$ which is the space of continuous $\Gamma_{\Phi, \lambda}$-valued functions on the interval $\left[0, t_{0}\right]$.

Girsanov's formula for finite systems. Let $\gamma_{0}^{n}$ be a sequence of finite measures from $\Gamma$ satisfying the condition:

$$
\gamma_{0}^{n} \rightarrow \gamma_{0}, \gamma_{0}^{n} \leq \gamma_{0}^{n+1}
$$

It was proved in [4] that under the condition (PC), for any $n$, there exists a unique strong solution to system (2) with the initial value $\gamma_{0}^{n}$. Denote it by $\gamma_{t}\left(\gamma_{0}^{n}, \omega\right)$. 
Lemma 1. Set $a\left(x, x^{\prime}\right)=a\left(x-x^{\prime}\right)$,

$$
\begin{gathered}
G_{1}^{n}(t)=\sigma^{-1} \sum_{x_{i} \in S_{\gamma_{0}^{n}}} \int_{0}^{t}\left(\left\langle a\left(x_{i}+\sigma w_{i}(s), .\right), \gamma_{s}^{*}\left(\gamma_{0}^{n}, \omega\right)\right\rangle, d w_{i}(s)\right), \\
G_{2}^{n}(t)=\sigma^{-2} \int_{0}^{t} \int\left|\left\langle a(x, .), \gamma_{s}^{*}\left(\gamma_{0}^{n}, \omega\right)\right\rangle\right|^{2} \gamma_{s}^{*}\left(\gamma_{0}^{n}, \omega, d x\right) d s \\
\rho_{n}(t)=\exp \left\{G_{1}^{n}(t)-\frac{1}{2} G_{2}^{n}(t)\right\} .
\end{gathered}
$$

Then

$$
\begin{gathered}
E \Phi\left(\left\langle\phi_{1}, \gamma_{t_{1}}\left(\gamma_{0}^{n}, \omega\right)\right\rangle, \ldots,\left\langle\phi_{k}, \gamma_{t_{k}}\left(\gamma_{0}^{n}, \omega\right\rangle\right)\right. \\
=E \rho_{n}(t) \Phi\left(\left\langle\phi_{1}, \gamma_{t_{1}}^{*}\left(\gamma_{0}^{n}, \omega\right)\right\rangle, \ldots,\left\langle\phi_{k}, \gamma_{t_{k}}^{*}\left(\gamma_{0}^{n}, \omega\right)\right\rangle\right)
\end{gathered}
$$

for all $k=1,2, \ldots$, bounded continuous functions $\Phi: R^{k} \rightarrow R$ and $\phi_{1}, \ldots, \phi_{k} \in$ $C_{f}, t_{1}, \ldots, t_{k} \in[0, t]$.

The proof of the lemma can be obtained using the approximation of the function $a\left(x, x^{\prime}\right)$ by smooth functions since for smooth $a\left(x, x^{\prime}\right)$ the proof is a consequence of Girsanov's formula [8].

Introduce the stochastic processes

$$
w_{k}^{c}(t)=\int_{0}^{t} 1_{\left\{\left|x_{k}+w_{k}(s)\right| \leq c\right\}} d w_{k}(s), \quad x_{k} \in S_{\gamma_{0}^{n}},
$$

where $c>0$ is a constant. Let $\mathcal{F}_{t}^{n, c}$ be the $\sigma$-algebra generated by

$$
\left\{w_{k}^{c}(s), s \leq t, x_{k} \in S_{\gamma_{0}^{n}}\right\} .
$$

\section{Lemma 2.}

$$
E\left(\rho_{n} / \mathcal{F}_{t}^{n, c}\right)=\rho_{n}(c, t)
$$

where

$$
\rho_{n}(c, t)=\exp \left\{G_{1}^{n}(c, t)-\frac{1}{2} G_{2}^{n}(c, t)\right\}
$$

and

$$
\begin{aligned}
& G_{1}^{n}(c, t)=\sigma^{-1} \sum_{x_{i} \in S_{\gamma_{0}^{n}}} \int_{0}^{t}\left(E\left(\left\langle a\left(x_{i}+\sigma w_{i}(s), .\right), \gamma_{s}^{*}\left(\gamma_{0}^{n}, \omega\right)\right\rangle / \mathcal{F}_{s}^{n, c}\right), d w_{i}^{c}(s)\right), \\
& G_{2}^{n}(c, t)=\sigma^{-2} \int_{0}^{t} \int\left|E\left(\left\langle a(x, .), \gamma_{s}^{*}\left(\gamma_{0}^{n}, \omega\right)\right\rangle / \mathcal{F}_{s}^{n, c}\right)\right|^{2} 1_{\{|x| \leq c\}} \gamma_{s}^{*}\left(\gamma_{0}^{n}, \omega, d x\right) d s .
\end{aligned}
$$

The proof rests on the statement below.

Statement. Let $\mathcal{F}_{t}, t \in R_{+}$be a continuous filtration, and its subfiltration $\mathcal{F}_{t}^{*}, t \in R_{+}$, satisfy the condition

$(\mathrm{RE}) E\left(\xi / \mathcal{F}_{t}\right)$ is a $\mathcal{F}_{t}^{*}$-measurable random variable if $\xi$ is a bounded $\mathcal{F}_{\infty}^{*}-$ measurable random variable. 
Let $\mu_{t}$ be an $\mathcal{F}_{t}$-martingale with the square characteristic $\langle\mu\rangle_{t}$ for which the stochastic process

is a martingale. Then

$$
\rho(t)=\exp \left\{\mu_{t}-\frac{1}{2}\langle\mu\rangle_{t}\right\}
$$

$$
E\left(\rho(t) / \mathcal{F}_{t}^{*}\right)=\exp \left\{\mu_{t}^{*}-\frac{1}{2}\left\langle\mu^{*}\right\rangle_{t}\right\},
$$

where $\mu_{t}^{*}=E\left(\mu_{t} / \mathcal{F}_{t}^{*}\right)$ is a martingale, and $\left\langle\mu^{*}\right\rangle_{t}$ is its square characteristic.

Proof. Set $\tilde{\mu}_{t}=\mu_{t}-\mu_{t}^{*}$. Condition (RE) implies the relation

$$
E\left(\int_{0}^{t} g(s) d \tilde{\mu}_{s} / \mathcal{F}_{\infty}^{*}\right)=0
$$

for all $\mathcal{F}_{t}$-adapted functions $g(t)$ for which

$$
E\left|\int_{0}^{t} g(s) d \tilde{\mu}_{s}\right|<\infty
$$

Set

$$
\rho^{*}(t)=\exp \left\{\mu_{t}^{*}-\frac{1}{2}\left\langle\mu^{*}\right\rangle_{t}\right\}, \quad \tilde{\rho}(t)=\exp \left\{\tilde{\mu}_{t}-\frac{1}{2}\langle\tilde{\mu}\rangle_{t}\right\},
$$

where $\langle\tilde{\mu}\rangle_{t}$ is the square characteristic of the martingale $\tilde{\mu}_{t}$. The proof follows from the relations $\rho(t)=\rho^{*}(t) \tilde{\rho}(t)$ and

$$
E\left(\tilde{\rho}(t)-1 / \mathcal{F}_{\infty}^{*}\right)=E\left(\int_{0}^{t} \tilde{\rho}(s) d \tilde{\mu}(s) / \mathcal{F}_{\infty}^{*}\right)=0 .
$$

Remark 1. Assume that $\phi_{i}(x)=0$ for $|x| \geq c, i=1,2, \ldots, k, \phi_{1}, \ldots, \phi_{k} \in$ $\mathcal{C}_{f}, t_{1}, \ldots, t_{k} \in[0, t]$. Then for $\Phi$ satisfying the conditions of Lemma 1 we have the relation

$$
\begin{gathered}
E \Phi\left(\left\langle\phi_{1}, \gamma_{t_{1}}\left(\gamma_{0}^{n}, \omega\right)\right\rangle, \ldots,\left\langle\phi_{k}, \gamma_{t_{k}}\left(\gamma_{0}^{n}, \omega\right)\right\rangle\right) \\
=E \rho_{n}(c, t) \Phi\left(\left\langle\phi_{1}, \gamma_{t_{1}}^{*}\left(\gamma_{0}^{n}, \omega\right)\right\rangle, \ldots,\left\langle\phi_{k}, \gamma_{t_{k}}^{*}\left(\gamma_{0}^{n}, \omega\right)\right\rangle\right) .
\end{gathered}
$$

Remark 2. Denote by $\mathcal{F}_{t}^{c}$ the $\sigma$-algebra generated by $\left\{w_{k}^{c}(s), s \leq t, x_{k} \in S_{\gamma_{0}}\right\}$. Then there exist the limits in probability

$$
\begin{aligned}
& \lim _{n \rightarrow \infty} G_{1}^{n}(c, t)=G_{1}(c, t), \quad \lim _{n \rightarrow \infty} G_{2}^{n}(c, t)=G_{2}(c, t), \\
& \lim _{n \rightarrow \infty} \rho_{n}(t)=\rho(c, t)=\exp \left\{G_{1}(c, t)-\frac{1}{2} G_{2}(c, t)\right\},
\end{aligned}
$$

where

$$
\begin{aligned}
& \left.G_{1}(c, t)=\sigma^{-1} \sum_{x_{i} \in S_{\gamma_{0}}} \int_{0}^{t}\left(E\left(\left\langle a\left(x_{i}+\sigma w_{i}(s), .\right), \gamma_{s}^{*}\left(\gamma_{0}, \omega\right)\right\rangle / \mathcal{F}_{s}^{c}\right), d w_{i}^{c}(s)\right)\right), \\
& G_{2}(c, t)=\sigma^{-2} \int_{0}^{t} \int\left|E\left(\left\langle a(x, .), \gamma_{s}^{*}\left(\gamma_{0}, \omega\right)\right\rangle / \mathcal{F}_{s}^{c}\right)\right|^{2} 1_{\{|x| \leq c\}} \gamma_{s}^{*}\left(\gamma_{0}, \omega, d x\right) d s .
\end{aligned}
$$


Let $w(t)$ be a standard Wiener process. Introduce the functions

$$
\begin{gathered}
Q_{c}(s, x, B)=P\left\{x+\sigma w(s) \in B, \inf _{u \leq s}|x+\sigma w(u)|>c\right\}, \\
x \in V_{c}, \quad B \in \mathcal{B}\left(V_{c}\right), V_{c}=\left\{x \in R_{d}:|x|>c\right\}, \\
Q_{c}^{*}(s, x, B)=\lim _{\lambda \downarrow 1} \frac{Q_{c}(s, x, B)}{Q_{c}\left(s, \lambda x, V_{c}\right)},|x|=c, \quad B \in \mathcal{B}\left(V_{c}\right) .
\end{gathered}
$$

Set

$$
\theta_{i}(c, s)=\inf \Delta_{i}(c, s), \quad \zeta_{i}(c, s)=\sup \Delta_{i}(c, s)
$$

where

$$
\Delta_{i}(c, s)=\left\{u \leq s:\left|x_{i}^{*}(u)\right| \leq c\right\}, x_{i}^{*}(u)=x_{i}+w_{i}(u) .
$$

Then the following statement holds.

\section{Lemma 3.}

$$
\begin{aligned}
& E\left(a\left(x, x_{i}^{*}(s)\right) 1_{\left\{\left|x_{i}^{*}(s)\right| \leq c\right\}} / \mathcal{F}_{s}^{c}\right)=a\left(x, x_{i}^{*}(s)\right) 1_{\left\{\left|x_{i}^{*}(s)\right| \leq c\right\}} \\
& +1_{\left\{\theta_{i}(c, s)<\infty\right\}} \int a(x, z) Q_{c}^{*}\left(s-\zeta_{i}(c, s), x_{i}^{*}\left(\zeta_{i}(c, s)\right), d z\right) \\
& +1_{\left\{\theta_{i}(c, s)=+\infty\right\}} \int a(x, z) Q_{c}\left(s, x_{i}, d z\right) .
\end{aligned}
$$

Corollary 1. Introduce the measures

$$
\begin{gathered}
\nu_{s}(A)=\sum_{k} 1_{\left\{x_{k} \in A\right\}} 1_{\left\{\theta_{k}(c, s)=+\infty\right\}}, \quad A \in \mathcal{B}\left(V_{c}\right), \\
\nu_{s}^{*}(\Lambda, A)=\sum_{k} 1_{\left\{\zeta_{k}(c, s) \in \Lambda\right\}} 1_{\left\{x_{k}\left(\zeta_{k}(c, s)\right) \in A\right\}}, \\
\Lambda \in \mathcal{B}([0, s]), \quad A \in \mathcal{B}\left(V_{c}^{\prime}\right), \quad V_{c}^{\prime}=\left\{x \in R_{d}:|x|=c\right\} .
\end{gathered}
$$

Then

$$
E\left(\left\langle a(x, \cdot), \gamma_{s}^{*}\left(\gamma_{0}, \omega\right)\right\rangle / \mathcal{F}_{s}^{c}\right)=a_{c}(s, x, \omega)+\int a\left(x, x^{\prime}\right) 1_{\left\{\left|x^{\prime}\right| \leq c\right\}} \gamma_{s}^{*}\left(\gamma_{0}, \omega, d x^{\prime}\right),
$$

where

$$
\begin{aligned}
& a_{c}(s, x, \omega)=\iint a(x, z) Q_{c}\left(s, x^{\prime}, d z\right) \nu_{s}\left(d x^{\prime}\right) \\
& +\iiint a(x, z) Q_{c}^{*}\left(s-u, x^{\prime}, d z\right) \nu_{s}^{*}\left(d u, d x^{\prime}\right) .
\end{aligned}
$$

Corollary 2. The functions $G_{k}(c, t), k=1,2$, can be represented in the form

$$
G_{1}(c, t)=\sigma^{-1} \int_{0}^{t} \sum_{i}\left(a_{c}\left(s, x_{i}^{*}(s), \omega\right), d w_{i}^{c}(s)\right),
$$

and

$$
G_{2}(c, t)=\sigma^{-2} \int_{0}^{t} H(c, s) d s,
$$


where

$$
H_{c}(s)=\sum_{i}\left|\sum_{j \neq i} a\left(x_{i}^{*}(s), x_{j}^{*}(s)\right) 1_{\left\{\left|x_{j}^{*}(s)\right| \leq c\right\}}+a_{c}\left(s, x_{i}^{*}(s), \omega\right)\right|^{2} 1_{\left\{\left|x_{i}^{*}(s)\right| \leq c\right\}} .
$$

Remark 3. Denote by $\left(\mathcal{F}_{t}^{c}(i)\right)$ the filtration generated by the stochastic process $w_{i}^{c}(t)$, and by $\left(\mathcal{F}_{t}^{c}(i, j)\right)$ the filtration generated by the pair of stochastic processes $\left(w_{i}^{c}(t) ; w_{j}^{c}(t)\right), i \neq j$. Set

$$
\rho^{i}(c, t)=E\left(\rho(c, t) / \mathcal{F}_{t}^{c}(i)\right), \quad \rho^{i, j}(c, t)=E\left(\rho(c, t) / \mathcal{F}_{t}^{c}(i, j) .\right.
$$

Then

$$
\rho^{i}(c, t)=\exp \left\{\int_{0}^{t}\left(g^{i}(c, s), d w_{i}^{c}(s)\right)-\frac{1}{2} \int_{0}^{t}\left|g^{i}(c, s)\right|^{2} d s\right\}
$$

where

$$
\sigma g^{i}(c, s)=\left[E\left(a_{c}(s, x)+\sum_{k \neq i} a\left(x, x_{k}^{*}(s)\right) 1_{\left\{\left|x_{k}^{*}(s)\right| \leq c\right\}}\right)\right]_{\left\{x=x_{i}^{*}(s)\right\}},
$$

and

$$
\begin{aligned}
\rho^{i, j}(c, t) & =\exp \left\{\int_{0}^{t}\left[\left(g_{i j}^{i}(c, s), d w_{i}^{c}(s)\right)+\left(g_{i j}^{j}(c, s), d w_{j}^{c}(s)\right)\right]\right\} \\
& =\exp \left\{-\frac{1}{2} \int_{0}^{t}\left[\left|g_{i j}^{i}(c, s)\right|^{2}+\left|g_{i j}^{j}(c, s)\right|^{2}\right] d s\right\},
\end{aligned}
$$

where

$$
\begin{gathered}
\sigma g_{i j}^{i}(c, s)=a\left(x_{i}^{*}(s), x_{j}^{*}(s)\right) 1_{\left\{\left|x_{i}^{*}(s)\right| \wedge\left|x_{j}^{*}(s)\right| \leq c\right\}} \\
+\left[E\left(a_{c}(s, x)+\sum 1_{\{k \neq i\}} 1_{\{k \neq j\}} a\left(x, x_{k}^{*}(s)\right) 1_{\left\{\left|x_{k}^{*}(s)\right| \leq c\right\}}\right)\right]_{\left\{x=x_{i}^{*}(s)\right\}}
\end{gathered}
$$

Remark 4. Assume that $\tau_{c}$ is a stopping time with respect to the filtration $\left(\mathcal{F}_{t}^{c}\right)_{t \geq 0}$ satisfying the condition $G_{2}\left(c, \tau_{c}\right) \leq c_{1}$, where $c_{1}$ is a constant. Then $E \rho\left(c, \tau_{c}\right)=1$ and $E\left(\rho\left(c, \tau_{c}\right)\right)^{2} \leq \exp \left\{c_{1}\right\}$.

Introduce the stochastic processes

$$
\begin{gathered}
w_{i}^{*}(c, t)=w_{i}(t)-\sigma^{-1} \int_{0}^{t \wedge \tau_{c}} a_{c}\left(s, x_{i}^{*}(s), \omega\right) 1_{\left\{\left|x_{i}^{*}(s)\right| \leq c\right\}} d s \\
-\sigma^{-1} \int_{0}^{t \wedge \tau_{c}} \sum_{i \neq j} a\left(x_{i}^{*}(s), x_{j}^{*}(s)\right) 1_{\left\{\left|x_{i}^{*}(s)\right| \vee\left|x_{j}^{*}(s)\right| \leq c\right\}} d s .
\end{gathered}
$$

Lemma 4. Denote by $\{\Omega, \mathcal{F}, P\}$, the probability space generated by the sequence $\left\{w_{k}(t), k=1,2, \ldots\right\}$ and let $P_{c}$ be the measure on $\{\Omega, \mathcal{F}\}$ for which

$$
\frac{d P_{c}}{d P}(\omega)=\rho\left(c, \tau_{c}\right) .
$$


Then $\left\{w_{k}(c, t), k=1,2, \ldots\right\}$ is the sequence of independent Wiener processes on the probability space $\left\{\Omega, \mathcal{F} . P_{c}\right\}$.

The proof is a consequence of Girsanov's results [8].

Remark 5. Let $c_{1}<c_{2}$ and $\tau_{c_{k}}$ be stopping time with respect to the filtration $\left(\mathcal{F}_{t}^{c_{k}}\right)_{t \geq 0}, k=1,2, \tau_{c_{1}}<\tau_{c_{2}}$ and $G_{2}\left(c_{1}, \tau_{c_{1}}\right)+G_{2}\left(c_{2}, \tau_{c_{2}}\right) \leq c_{3}$, where $c_{3}$ is a constant. Then

$$
E\left(\rho\left(c_{2}, \tau_{c_{2}}\right) / \mathcal{F}_{\tau_{c_{1}}}^{c_{1}}\right)=\rho\left(c_{1}, \tau_{c_{1}}\right)
$$

This formula is a consequence of the relation

$$
E\left(G_{1}\left(c_{2}, \tau_{c_{2}}\right) / \mathcal{F}_{\tau_{c_{1}}}^{c_{1}}\right)=G_{1}\left(c_{1}, \tau_{c_{1}}\right) .
$$

Lemma 5. Let $\left\{c_{k}, k=1,2, \ldots\right\}$ be a sequence of positive numbers, for which $\lim _{k \rightarrow+\infty} c_{k}=+\infty$. Then there exists a sequence of positive numbers $\left\{a_{k}\right\}$ for which

$$
P\left\{\sum_{k} a_{k} G_{2}\left(c_{k}, t\right)<\infty\right\}=1
$$

for all $t>0$.

Proof. Choose $a_{k}$ satisfying the inequality

$$
P\left(G_{2}\left(c_{k}, k\right)>\left(k^{2} a_{k}\right)^{-1}\right)<k^{-2} .
$$

Then for any $t>0$ we have the relation

$$
\sum_{k} P\left(a_{k} G_{2}\left(c_{k}, t\right)>k^{-2}\right)<t+\sum_{k \geq t} k^{-2}<\infty
$$

This completes the proof.

Corollary 3. Let a sequence $\left\{a_{k}\right\}$ satisfy the statement of Lemma 5. Set

$$
G(t)=\sum_{k} a_{k} G_{2}\left(c_{k}, t\right)
$$

With probability $1 G(t)$ is an increasing continuous function satisfying the relations $G(0)=0, \lim _{t \rightarrow \infty} G(t)=\infty$. Set

$$
\tau^{*}=\inf \{t: G(t)>b\}
$$

where $b$ is a positive number. Then

$$
E \rho\left(c_{k}, \tau^{*}\right)=1, \quad E\left(\rho\left(c_{k}, \tau^{*}\right)\right)^{2} \leq \exp \left\{b a_{k}^{-1}\right\} .
$$




\section{A Theorem on Existence of a Local Weak Solution}

Theorem 1. Let conditions (PC) and (IC) be fulfillrd, and let $\tau^{*}$ be a stopping time introduced by relation (9). Then the following statements hold:

(i) there exists a probability measure $P^{*}$ on $(\Omega, \mathcal{F})$ for which

$$
P^{*}(A)=\lim _{c \rightarrow \infty} E 1_{A} \rho(c, \tau *), \quad A \in \bigvee_{k} \mathcal{F}_{\tau^{*}}^{c_{k}}, \quad P^{*}(A)=P(A), A \in \mathcal{F}^{*},
$$

where the $\sigma$-algebra $\mathcal{F}^{*}$ is generated by the processes

$$
\left\{x_{k}^{*}\left(t \vee \tau^{*}\right)-x_{k}^{*}(t), \quad t \geq 0, \quad k=1,2, \ldots\right\} .
$$

(ii) the stochastic processes given by the formula

$$
\sigma w_{k}^{*}(t)=x_{k}^{*}(t)-\int_{0}^{t \wedge \tau^{*}} \sum_{i \neq k} a\left(x_{k}^{*}(s), x_{i}^{*}(s)\right) d s, \quad k=1,2, \ldots,
$$

are independent Wiener processes with respect to the filtration $\left(\mathcal{F}_{t}\right)_{(t \geq 0)}$ on the probability space $\left(\Omega, \mathcal{F}, P^{*}\right)$.

Proof. Since $\tau^{*}$ is a stopping time, for $A \in \mathcal{F}_{\tau^{*}}^{c_{k}}$ we can write, using Remark 5, the relations

$$
E 1_{A} \rho\left(c, \tau^{*}\right)=E 1_{A} \rho\left(c_{k}, \tau^{*}\right)
$$

if $c>c_{k}$. This implies the existence of limits

$$
\lim _{c \rightarrow \infty} 1_{A \times B} \rho\left(c, \tau^{*}\right), \quad A \in \bigvee_{k} \mathcal{F}_{\tau^{*}}^{c_{k}}, \quad B \in \mathcal{F}^{*} .
$$

It follows from the last formula that there exists a limit

$$
\lim _{c \rightarrow \infty} \Phi\left(\xi_{1}, \ldots, \xi_{m}\right) \rho\left(c, \tau^{*}\right),
$$

where $\Phi \in \mathcal{C}\left(R^{m}\right)$ and

$$
\xi_{i}=\left\langle f_{i}, \gamma_{t_{i}}^{*}\left(\gamma_{0}, \omega\right)\right\rangle, \quad i=1, \ldots, m,
$$

where $f_{i} \in \mathcal{C}\left(R^{d}\right), f_{i}(x)=0$ for $|x|$ large enough.

Now we prove that there exists a probability measure $P^{*}$ on $(\Omega, \mathcal{F})$ for which a limit in the formula $(10)$ is represented in the form $E^{*} \Phi\left(\xi_{1}, \ldots, \xi_{m}\right)$, where $E^{*}$ is the expectation with respect to the probability $P^{*}$. Set

$$
f_{n}\left(x, x^{\prime}\right)=\left(1-\frac{|x|}{c_{n}}\right)\left(1-\frac{\left|x^{\prime}\right|}{c_{n}}\right)\left(1+\frac{1}{\left|x-x^{\prime}\right|}\right) \vee 0 .
$$

Using Corollary 3 we can write the inequality

$$
\begin{gathered}
E\left\langle f_{n}, \gamma_{t}^{*}\left(\gamma_{0}, \omega\right) \times \gamma_{t}^{*}\left(\gamma_{0}, \omega\right)\right\rangle \rho\left(c, \tau^{*}\right) \\
\leq\left(E\left\langle f_{n}, \gamma_{t}^{*}\left(\gamma_{0}, \omega\right) \times \gamma_{t}^{*}\left(\gamma_{0}, \omega\right)\right\rangle^{2}\right)^{\frac{1}{2}} \exp \left\{\frac{1}{2} b a_{n}^{-1}\right\} .
\end{gathered}
$$

Denote

$$
A_{n}(t)=E\left\langle f_{n}, \gamma_{t}^{*}\left(\gamma_{0}, \omega\right) \times \gamma_{t}^{*}\left(\gamma_{0}, \omega\right)\right\rangle^{2}
$$


The functions $A_{n}(t)$ are non-negative and continuous. There exists a sequence of positive numbers $\left\{b_{n}\right\}$ for which

$$
\sum_{n} b_{n}\left(1+A_{n}(t)\right) \exp \left\{\frac{1}{2} b a_{n}^{-1}\right\}<\infty, \quad t>0 .
$$

Formulas (11) and (12) imply the relation

$$
\limsup _{c \rightarrow \infty} E \sum_{n} b_{n}\left\langle f_{n}, \gamma_{t}^{*}\left(\gamma_{0}, \omega\right) \times \gamma_{t}^{*}\left(\gamma_{0}, \omega\right)\right\rangle \rho\left(c, \tau^{*}\right)<\infty
$$

This formula implies that the set of measures $\left\{P_{c}^{*}, c>0\right\}$ for which

$$
\frac{d P_{c}^{*}}{d P}(\omega)=\rho\left(c, \tau^{*}\right)
$$

is weakly compact, so there exists a sequence $c_{k}^{\prime}, c_{k}^{\prime} \rightarrow \infty$ for which $P_{c_{k}^{\prime}}^{*}$ converges weakly to a measure $P^{*}$. The statement (i) is proved.

Introduce the stopping times

$$
\tau_{n}^{*}=\inf \left\{t: \sum_{k \leq n} a_{k} G_{2}\left(c_{k}, t\right) \geq b\right\},
$$

and set

$$
P_{n}^{*}(A)=E 1_{A} \rho\left(c_{n}, \tau_{n}^{*}\right), \quad A \in \mathcal{F} .
$$

Let $\Phi\left(\xi_{1}, \ldots, \xi_{m}\right)$ be the same as before. Then

$$
\lim _{n \rightarrow \infty} E_{n}^{*} \Phi\left(\xi_{1}, \ldots, \xi_{m}\right)=E^{*} \Phi\left(\xi_{1}, \ldots, \xi_{m}\right) ;
$$

here $E_{n}^{*}$ is the expectation with respect to probability $P_{n}^{*}$. This formula is a consequence of the relations

$$
\tau_{n}^{*}>\tau^{*}, \quad \tau_{n}^{*} \rightarrow \tau^{*}
$$

in probability and

$$
E\left(\rho\left(c_{n}, \tau_{n}^{*}\right) / \mathcal{F}_{\tau^{*}}^{c_{n}}\right)=\rho\left(c_{n}, \tau *\right) .
$$

Let $w_{i}^{*}(c, t)$ be given by formula $(8)$. Then for fixed $n$ the sequence

$$
\left\{w_{i}^{*}\left(c_{n}, t\right), i=1,2, \ldots\right\}
$$

represents independent Wiener processes on the probability space $\left\{\Omega, \mathcal{F}, P_{n}^{*}\right\}$. Introduce stopping times

$$
\zeta_{i}^{n}=\inf \left\{t:\left|x_{i}(t)\right|>c_{n}-r\right\} .
$$

Since $a_{c}(x, \omega)=0$ for $|x|<c_{n}-r$,we have

$$
w_{i}^{*}\left(c_{n}, t \vee \zeta_{i}^{n}\right)=w_{i}^{*}\left(t \vee \zeta_{i}^{n}\right)
$$

Using Remark 3 we can prove that the relation

$$
\lim _{c \rightarrow \infty} \limsup _{n \rightarrow \infty} P_{n}^{*}\left\{\sup _{s \leq t}\left|x_{i}^{*}(s)\right|>c\right\}=0
$$


is fulfilled for any $i$ and $t>0$. Let $\Phi, h_{1}, \ldots, h_{m}$ be the same as before. Set

$$
\xi_{i}^{*}=\int_{0}^{t} h_{i}(s) d w_{i}^{*}(s), \quad \xi_{i}^{n}=\int_{0}^{t} h_{i}(s) d w_{i}^{*}\left(c_{n}, s\right), \quad i=1, \ldots .
$$

Note that

$$
E_{n}^{*} \Phi\left(\xi_{1}^{n}, \ldots, \xi_{m}^{n}\right)=E \Phi\left(\int_{0}^{t} h_{1}(s) d w_{1}(s), \ldots, \int_{0}^{t} h_{m}(s) d w_{m}(s)\right)
$$

for all $n$. Denote the expression in the right hand side of formula (14) by $\bar{\Phi}$. Then

$$
E_{n}^{*} \Phi\left(\xi_{1}^{*}, \ldots, \xi_{m}^{*}\right)=\bar{\Phi}+E_{n}^{*} O\left(\sum_{i \leq m} 1_{\left\{\sup _{s \leq t}\left|x_{i}^{*}(s)\right|>c_{n}-r\right\}}\right) .
$$

Formulas (13) and (15) imply the relation

$$
E^{*} \Phi\left(\xi_{1}^{*}, \ldots, \xi_{m}^{*}\right)=\lim _{n \rightarrow \infty} E_{n}^{*} \Phi\left(\xi_{1}^{*}, \ldots, \xi_{m}^{*}\right)=\bar{\Phi} .
$$

The statement (ii) is proved.

\section{REFERENCES}

1. S. Albeverio, Yu. G. Kondratiev, and Röckner, Analysis and geometry on configuration spaces. J. Funct. Anal. 154(1998), 444-500.

2. S. Albeverio, Yu. G. Kondratiev, and Röckner, Analysis and geometry on configuration spaces: the Gibbsian case. J. Funct. Anal. 157(1998), 242-291.

3. A. V. Skoroknod, Stochastic equations for a complex system. D. Reidel Publishing Co., Dordrecht-Boston, Mass., 1988.

4. A. V. Skorokнod, On the regularity of many-particle dynamical systems perturbed by white noise. J. Appl. Math. Stoch. Anal. 9(1996), 427-437.

5. Yu. L. DALETSKII, Infinite-dimensional elliptic operators and related parabolic equations. (Russian) Uspekhi. Mat. Nauk 22(1967), 3-54.

6. J. Fritz, Gradient dynamics of infinite point systems. Ann. Probab. 15(1987), 478-514.

7. A. V. Skorokhod, On infinite systems of stochastic differential equations. Methods Funct. Anal. Topology (1999), No. 4, 54-61.

8. I. V. Girsanov, On a transformation of some class of stochastic processes by absolute continuous changing of measures. Theory Probab. Appl. 5(1960), 314-330.

(Received 3.07.2000)

Author's addresses:

Institute of Mathematics

National Academy of Sciences of Ukraine

3, Tereshchenkivska St., Kyiv 01601, Ukraine

Department of Statistics and Probability

Michigan State University

A 415 Wells Hall, East Lansing, MI 48824, U.S.A.

E-mail: skorokhod@stt.msu.edu 